\title{
Oscillation Criteria of Second-order Half-linear Neutral Dynamic Equations with Distributed Deviating Arguments
}

\author{
Yunhui Zeng \\ College of Mathematics and Statistics, Hengyang Normal University, Hunan, 421002 ,China \\ chj8121912@sina.com
}

Keywords: Oscillation; Dynamic equation; Distributed deviating arguments

Abstract: In this paper, we investigate the oscillation of second-order half-linear neutral dynamic equations with distributed deviating arguments. By using the generalized Riccati transformation and the inequality technique, we establish some new oscillatory criteria for all solutions to second-order half-linear neutral dynamic equations being oscillatory on the time scale .

\section{Introduction}

In this paper, we concern with the oscillation of second-order half-linear neutral dynamic equation with distributed deviating arguments of the form

$$
\left(r(t)\left|z^{\Delta}(t)\right|^{\alpha-1} z^{\Delta}(t)\right)^{\Delta}+\int_{a}^{b} q(t, \xi)|x(g(t, \xi))|^{\alpha-1} x(g(t, \xi)) \Delta \xi=0, t \in \mathrm{T},
$$

where $z(t)=x(t)+p(t) x(\tau(t))$ and $\alpha$ is a constant with $\alpha \geq 1$.

Throughout this paper, we always assume that

$\left(A_{1}\right) \quad r(t), p(t), \tau(t)$ are real-valued rd-continuous positive functions on time scales with $0 \leq p(t)<1, \tau(t) \leq t$ and $\lim _{t \rightarrow \infty} \tau(t)=\infty$, where $p(t)$ is increasing.

$\left(A_{2}\right) q(t, \xi) \in C_{r d}\left(\mathrm{~T} \times[a, b]_{\mathrm{T}},(0, \infty)\right)$ is not eventually identical zero and decreasing with respect to the second variable $\xi$, and $g(t, \xi) \in C_{r d}\left(\mathrm{~T} \times[a, b]_{\mathrm{T}}, \mathrm{T}\right)$ is strictly positive rd-continuous $\Delta$-differentiable on $\mathrm{T} \times[a, b]_{\mathrm{T}}$ with respect to the first variable $t$ and decreasing with respect to the second variable $\xi$, respectively, where $\xi \in[a, b]_{\mathrm{T}},[a, b]_{\mathrm{T}}=\{t \in \mathrm{T}: a \leq t \leq b\}$ and $\liminf _{t \rightarrow \infty, \xi \in[a, b]_{\mathrm{T}}}\{g(t, \xi)\}=\infty$.

$\left(A_{3}\right) \int_{t_{0}}^{\infty}\left(\frac{1}{r(t)}\right)^{\frac{1}{\alpha}} \Delta t=\infty$.

For a nontrivial solution $x(t)$ to the equation (1.1), we assume that $x(t)+p(t) x(\tau(t)) \in C_{r d}^{1}\left(\left[t_{y}, \infty\right)_{\mathrm{T}}\right)$ and $r(t)[x(t)+p(t) x(\tau(t))]^{\Delta} \in C_{r d}^{1}\left(\left[t_{y}, \infty\right)_{\mathrm{T}}\right)$, where $t \in\left[t_{0}, \infty\right)_{\mathrm{T}}$.

We restrict our attention to nontrivial solutions to equation (1.1) which exist on some half-line $\left[t_{y}, \infty\right)_{\mathrm{T}}$, and satisfy $\operatorname{Sup}\left\{|x(t)|: t \in\left[t_{1}, \infty\right)_{\mathrm{T}}\right\}>0$ for all $t_{1} \in\left[t_{y}, \infty\right)_{\mathrm{T}}$. Consequently, we will always assume that solutions to (1.1) exist for all $t_{0} \geq 0$. A solution $x(t)$ to (1.1) is called oscillatory if it is neither eventually positive nor eventually negative; otherwise it is called a non-oscillatory solution. The equation itself is called oscillatory if all its solutions are oscillatory. Since we are interested in oscillatory behaviour of solutions, we will suppose that the time scale T under consideration is not bounded, that is, it is a time scale interval of the form $\left[t_{0}, \infty\right)_{\mathrm{T}}$.

We note that if $\mathrm{T}=R$, we have $\sigma(t)=t, \mu(t)=0, z^{\Delta}(t)=z^{\prime}(t)$ and therefore (1.1) becomes a second-order half-linear neutral differential equation with distributed deviating arguments of the form

$$
\left(r(t)\left|z^{\prime}(t)\right|^{\alpha-1} z^{\prime}(t)\right)^{\prime}+\int_{a}^{b} q(t, \xi)|x(g(t, \xi))|^{\alpha-1} x(g(t, \xi)) d \xi=0 .
$$


If $\mathrm{T}=\mathrm{N}$, then $\sigma(t)=t+1, \mu(t)=1, z^{\Delta}(t)=\Delta z(t)=z(t+1)-z(t)$, and therefore (1.1) becomes a second-order half-linear neutral differential equation with distributed deviating arguments of the form $\Delta\left(r(t)|\Delta z(t)|^{\alpha-1} \Delta z(t)\right)+\sum_{\xi=a}^{b-1} q(t, \xi)|x(g(t, \xi))|^{\alpha-1} x(g(t, \xi))=0$.

On the other hand, if $\mathrm{T}=\mathrm{hN}$ for $\mathrm{h}>0$, then $\sigma(t)=t+h, \mu(t)=h, z^{\Delta}(t)=\Delta_{h} z(t)=\frac{z(t+h)-z(t)}{h}$, and therefore (1.1) becomes a second-order half-linear neutral differential equation with distributed deviating arguments of the form

$$
\Delta_{h}\left(r(t)\left|z_{h}^{\Delta}(t)\right|^{\alpha-1} z_{h}^{\Delta}(t)\right)+\sum_{\mathrm{k}=\frac{\mathrm{a}}{\mathrm{h}}}^{\frac{b}{h}-1} q(t, k h)|x(g(t, k h))|^{\alpha-1} x(g(t, k h))=0 .
$$

In recent years, the oscillation theory and the applications of dynamic equations on time scales have attracted much attenction (cf. [1-4,6). For nonlinear neutral dynamic equations, see [3,4,7]. However, the study of oscillatory criteria on dynamic equations with distributed deviating arguments is relatively less (cf. [3]). In this paper, by using the generalized Riccati transformation and the inequality technique, we establish some new oscillatory criteria for all solutions to second-order half-linear neutral dynamic equations being oscillatory on the time scale $\mathrm{T}$.

\section{Main Results}

We use the following notations for simplicity:

$$
\begin{gathered}
Q(t)=(b-a) q(t, b)(1-p(g(t, a)))^{\alpha}, \delta(t)=g(t, b), z^{[1]}(t)=r(t)\left|z^{\Delta}(t)\right|^{\alpha-1} z^{\Delta}(t), \quad z^{[2]}(t)=\left(z^{[1]}(t)\right)^{\Delta}, \\
\int_{T}^{t}\left(\frac{1}{r(s)}\right)^{1 / \alpha} \Delta s=r\left(t, T_{0}\right), \quad P_{2}(t)=Q(t)(\beta(t))^{\alpha}, \frac{1}{\beta(t)}=\frac{\int_{T}^{t}(r(s))^{-\frac{1}{\alpha}} \Delta s+\mu(t)(r(t))^{-\frac{1}{\alpha}}}{\int_{T}^{\delta(t)}(r(s))^{-\frac{1}{\alpha}} \Delta s}, \text { where } T_{0} \geq t_{0} \text { for }
\end{gathered}
$$

some sufficiently large number $t_{0}$.

Theorem2.1 Suppose that $\left(A_{k}\right)$ hold, where $k=1,2,3$. In addition, if

$$
\delta(t)>t \text { and } \int_{t_{0}}^{\infty} P_{1}(s) \Delta s=\infty \text { or } \delta(t) \leq t \text { and } \int_{t_{0}}^{\infty} P_{2}(s) \Delta s=\infty .
$$

Then every solution to equation (1.1) is oscillatory on $\left[t_{0}, \infty\right)_{\mathrm{T}}$.

Proof. Suppose not. Without loss of generality, we may assume that $x(t)$ is an eventually positive solution to equation (1.1) with $x(t)>0, x(\tau(t))>0$ and $x(g(t, \xi))>0$ for all $t \in\left[t_{1}, \infty\right)_{\mathrm{T}}$ and $\xi \in[a, b]_{\mathrm{T}}$. Then $z(t)=x(t)+p(t) x(\tau(t))>0$ for all $t \in\left[t_{1}, \infty\right)_{\mathrm{T}}$, where $t_{1} \geq t_{0}$. In view of equation (1.1), we have $\left(r(t)\left|z^{\Delta}(t)\right|^{\alpha-1} z^{\Delta}(t)\right)^{\Delta} \leq 0$, which implies $r(t)\left|z^{\Delta}(t)\right|^{\alpha-1} z^{\Delta}(t)$ is an eventually decreasing function. We claim that $r(t)\left|z^{\Delta}(t)\right|^{\alpha-1} z^{\Delta}(t)$ is eventually nonnegative on $\left[t_{1}, \infty\right)_{\mathrm{T}}$. If not, then there is a $t_{2} \geq t_{1}$ such that $r\left(t_{2}\right)\left|z^{\Delta}\left(t_{2}\right)\right|^{\alpha-1} z^{\Delta}\left(t_{2}\right)=c^{*}<0$, which yields $r(t)\left|z^{\Delta}(t)\right|^{\alpha-1} z^{\Delta}(t) \leq r\left(t_{2}\right)\left|z^{\Delta}\left(t_{2}\right)\right|^{\alpha-1} z^{\Delta}\left(t_{2}\right)=\mathrm{c}^{*}$

for $t>t_{2}$.

It follows that

$$
z^{\Delta}(t) \leq-\frac{\left(-c^{*}\right)^{\frac{1}{\alpha}}}{r^{\frac{1}{\alpha}}(t)} .
$$

Integrating (2.1) both sides from $t_{2}$ to $t$ and using $\left(A_{3}\right)$, we obtain 


$$
z(t) \leq z\left(t_{2}\right)-\left(-c^{*}\right)^{\frac{1}{\alpha}} \int_{t_{2}}^{t}\left(\frac{1}{r(s)}\right)^{\frac{1}{\alpha}} \Delta s \rightarrow-\infty
$$

as $t \rightarrow \infty$, which gives $z(t) \rightarrow-\infty$ as $t \rightarrow \infty$. This is a contradiction with the fact that $z(t)>0$ for all $t \in\left[t_{1}, \infty\right)_{\mathrm{T}}$.

Hence $r(t)\left|z^{\Delta}(t)\right|^{\alpha-1} z^{\Delta}(t) \geq 0$ eventually. Therefore, one concludes that there is a $t \geq t_{2} \geq t_{1}$ such that

$$
z(t)>0, z^{\Delta}(t)>0, z^{[1]}(t)>0, z^{[2]}(t)<0, t \geq t_{2}
$$

By (2.2) and $z(t)=x(t)+p(t) x(\tau(t))$, we have

$$
x(t) \geq z(t)-p(t) z(\tau(t)) \geq(1-p(t)) z(t), t \geq t_{2},
$$

which implies

$$
q(t, \xi) x^{\alpha}(g(t, \xi)) \geq q(t, b)(1-p(g(t, \xi)))^{\alpha} z^{\alpha}(g(t, \xi)) .
$$

Since $p(t)$ is increasing, by integrating (2.4) both sides from a to b, we have

$$
\int_{a}^{b} q(t, \xi) x^{\alpha}(g(t, \xi)) \Delta \xi \geq \int_{a}^{b} q(t, b)(1-p(g(t, \xi)))^{\alpha} z^{\alpha}(g(t, \xi)) \Delta \xi \geq Q(t) z^{\alpha}(\delta(t)) .
$$

Applying (2.5) to (1.1), we obtain

$$
\left(r(t)\left(x^{\Delta}(t)\right)^{\alpha}\right)^{\Delta}+Q(t) z^{\alpha}(\delta(t)) \leq 0 .
$$

Using (2.2) and the Potzche chain rule, for $\alpha \geq 1$, we get

$$
\left(z^{\alpha}(t)\right)^{\Delta}=\alpha \int_{0}^{1}\left[z(t)+h \mu(t) z^{\Delta}(t)\right]^{\alpha-1} d h z^{\Delta}(t) \geq \alpha \int_{0}^{1}(z(t))^{\alpha-1} d h z^{\Delta}(t)=\alpha(z(t))^{\alpha-1} z^{\Delta}(t)>0 .
$$

From (2.2), (2.6) and (2.7), we know that

$z^{[2]}(\mathrm{t})=\left(r(t)\left|z^{\Delta}(t)\right|^{\alpha-1} z^{\Delta}(t)\right)^{\Delta} \leq-(b-a) q(t, b)(1-p(g(t, a)))^{\alpha} z^{\alpha}(\delta(t))=-Q(t) z^{\alpha}(\delta(t)), t \geq t_{2}$.

For $t \geq t_{2}$, let

$$
w(t)=\frac{z^{[1]}(t)}{z^{\alpha}(t)} .
$$

It is obvious that $w(t)>0$, Taking the derivative of $w(t)$, we see that

$$
w^{\Delta}(t)=\frac{(z(\delta(t)))^{\alpha} z^{[2]}(t)}{(z(\delta(t)))^{\alpha}\left(z^{\sigma}(t)\right)^{\alpha}}-\frac{z^{[1]}(t)\left(z^{\alpha}(t)\right)^{\Delta}}{\left(z^{\sigma}(t)\right)^{\alpha} z^{\alpha}(t)} .
$$

Applying (2.8) to (2.10), we have

$$
w^{\Delta}(t) \leq-Q(t) \frac{z^{\alpha}(\delta(t))}{\left(z^{\sigma}(t)\right)^{\alpha}}-\frac{z^{[1]}(t)\left(z^{\alpha}(t)\right)^{\Delta}}{\left(z^{\sigma}(t)\right)^{\alpha} z^{\alpha}(t)} .
$$

On the other hand, using (2.7), we obtain

$$
\frac{\alpha z^{[1]}(t)(z(t))^{\alpha-1} z^{\Delta}(t)}{\left(z^{\sigma}(t)\right)^{\alpha} z^{\alpha}(t)}=\frac{\alpha z^{[1]}(t) z^{\Delta}(t)}{\left(z^{\sigma}(t)\right)^{\alpha} z(t)}=\frac{\alpha z^{[1]}(t)\left(z^{[1]}(t)\right)^{\frac{1}{\alpha}}}{\left(z^{\sigma}(t)\right)^{\alpha} z(t)(r(t))^{1 / \alpha}} \geq \frac{\alpha\left(1+w^{\sigma}(t)\right)^{1+\frac{1}{\alpha}}}{(r(t))^{1 / \alpha}}, t \geq t_{2} .
$$

Applying (2.12) to (2.11), we find

$$
w^{\Delta}(t) \leq-Q(t) \frac{z^{\alpha}(\delta(t))}{\left(z^{\sigma}(t)\right)^{\alpha}}-\frac{\alpha\left(1+w^{\sigma}(t)\right)^{1+\frac{1}{\alpha}}}{(r(t))^{1 / \alpha}}, t \geq t_{2} .
$$

(i) Let $\delta(t)>t$. Since $z^{[1]}(t)$ is decreasing, we see that

$$
z(t)=z\left(T_{0}\right)+\int_{T_{0}}^{t}\left(z^{[1]}(s)\right)^{1 / \alpha}\left(\frac{1}{r(s)}\right)^{1 / \alpha} \Delta s>\left(z^{[1]}(t)\right)^{1 / \alpha} \int_{T_{0}}^{t}\left(\frac{1}{r(s)}\right)^{1 / \alpha} \Delta s .
$$

Hence, the last inequality implies that

$$
\frac{z(t)}{\left(z^{[1]}(t)\right)^{1 / \alpha}}>\int_{T_{0}}^{t}\left(\frac{1}{r(s)}\right)^{1 / \alpha} \Delta s=r\left(t, T_{0}\right), t \geq T_{0} \geq t_{2} .
$$


Moreover, by using $z^{\sigma}(t)=z(t)+\mu(t) z^{\Delta}(t),\left(H_{2}\right)$ and (2.15), we have

$$
\frac{z^{\sigma}(t)}{z(t)}=\frac{z(t)+\mu(t) z^{\Delta}(t)}{z(t)}=1+\mu(t) \frac{z^{\Delta}(t)}{z(t)}=1+\frac{\mu(t)\left(z^{[1]}(t)\right)^{1 / \alpha}}{(r(t))^{1 / \alpha} z(t)} \leq \frac{\mu(t)+(r(t))^{\frac{1}{\alpha}} r\left(t, T_{0}\right)}{(r(t))^{1 / \alpha} r\left(t, T_{0}\right)},
$$

which is equivalent to

$$
\frac{z(t)}{z^{\sigma}(t)} \geq \frac{(r(t))^{\frac{1}{\alpha}} r\left(t, T_{0}\right)}{(r(t))^{1 / \alpha} r\left(t, T_{0}\right)+\sigma(t)-t} .
$$

Moreover, by using (2.16), we conclude that

$$
\frac{z(\delta(t))}{z^{\sigma}(t)}=\frac{z(\delta(t)) z(t)}{z(t) z^{\sigma}(t)} \geq \frac{z(\delta(t))}{z(t)} \cdot \frac{(r(t))^{1 / \alpha} r\left(t, T_{0}\right)}{(r(t))^{1 / \alpha} r\left(t, T_{0}\right)+\sigma(t)-t} .
$$

Since $\delta(t)>t$ and $z(t)$ is increasing, we know $z(\delta(t))>z(t)$.

(2.17) and (2.18) yiled

$$
\left(\frac{z(\delta(t))}{z^{\sigma}(t)}\right)^{\alpha} \geq\left(\frac{z(\delta(t))}{z(t)}\right)^{\alpha}\left(\frac{(r(t))^{1 / \alpha} r\left(t, T_{0}\right)}{(r(t))^{1 / \alpha} r\left(t, T_{0}\right)+\sigma(t)-t}\right)^{\alpha} \geq\left(\frac{(r(t))^{1 / \alpha} r\left(t, T_{0}\right)}{(r(t))^{1 / \alpha} r\left(t, T_{0}\right)+\sigma(t)-t}\right)^{\alpha} .
$$

Applying (2.19) to (2.13), we obtain

$$
w^{\Delta}(t) \leq-P_{1}(t)-\alpha \frac{\left(w^{\sigma}(t)\right)^{1+\frac{1}{\alpha}}}{(r(t))^{\frac{1}{\alpha}}}, t \geq T_{0} .
$$

On the other hand, as (2.15) using the definition of $z^{[1]}(t)$,we obtain

$$
w(t)=\frac{z^{[1]}(t)}{z^{\alpha}(t)} \leq\left(\int_{T_{0}}^{t}\left(\frac{1}{r(s)}\right)^{1 / \alpha} \Delta s\right)^{-\alpha}, t \geq T_{0} .
$$

By the last inequality and $\left(A_{3}\right)$, we conclude that

$$
\lim _{t \rightarrow \infty} w(t)=0
$$

Integrating (2.20) from $T_{0}$ to $t$, we have

$$
w(t) \leq w\left(T_{0}\right)-\int_{T_{0}}^{t} P_{1}(s) \Delta s-\alpha \int_{T_{0}}^{t} \frac{\left(w^{\sigma}(s)\right)^{1+\frac{1}{\alpha}}}{(r(s))^{\frac{1}{\alpha}}} \Delta s,
$$

using (2.0) and (2.21), which give $w\left(T_{0}\right) \geq \int_{T_{0}}^{\infty} P_{1}(s) \Delta s=\infty$.

This is a contradiction and the proof of part (i) is complete.

(ii) Let $\delta(t) \leq t$. Since $z^{[1]}(t)$ is decreasing, as in part (i), we see that

$$
z^{\Delta}(t)=\frac{\left(z^{[1]}(t)\right)^{1 / \alpha}}{(r(t))^{1 / \alpha}} .
$$

Integrating (2.22) both sides from $\delta(t)$ to $\sigma(t)$, we have

$$
z^{\sigma}(t)-z(\delta(t))=\int_{\delta(t)}^{\sigma(t)} \frac{\left[z^{[1]}(s)\right]^{1 / \alpha}}{(r(s))^{1 / \alpha}} \Delta s \leq\left(z^{[1]}(\delta(t))\right)^{1 / \alpha} \int_{\delta(t)}^{\sigma(t)} \frac{1}{(r(s))^{1 / \alpha}} \Delta s,
$$

which is equivalent to

$$
\frac{z^{\sigma}(t)}{z(\delta(t))} \leq 1+\frac{\left(z^{[1]}(\delta(t))\right)^{1 / \alpha}}{z(\delta(t)} \int_{\delta(t)}^{\sigma(t)} \frac{1}{(r(s))^{1 / \alpha}} \Delta s
$$


Moreover, $z(\delta(t))>z(\delta(t))-z\left(T_{0}\right) \geq\left(z^{[1]}(\delta(t))\right)^{1 / \alpha} \int_{T_{0}}^{\delta(t)} \frac{1}{(r(s))^{1 / \alpha}} \Delta s$, which is equivalent to

$$
\frac{\left[z^{[1]}(\delta(t))\right]^{\frac{1}{\alpha}}}{z(\delta(t))} \leq\left(\int_{T_{0}}^{\delta(t)} \frac{1}{(r(s))^{1 / \alpha}} \Delta s\right)^{-1} .
$$

Applying (2.24) to (2.23) and using the fact that $\int_{t}^{\sigma(t)} f(s) \Delta s=\mu(t) f(t)$, we obtain

$$
\frac{z^{\sigma}(t)}{z(\delta(t))}<1+\frac{\int_{\delta(t)}^{\sigma(t)}\left(\frac{1}{r(s)}\right)^{1 / \alpha} \Delta s}{\int_{T_{0}}^{\delta(t)}\left(\frac{1}{r(s)}\right)^{1 / \alpha} \Delta s}=\frac{\int_{T_{0}}^{t}\left(\frac{1}{r(s)}\right)^{1 / \alpha} \Delta s+\mu(t)(r(t))^{-1 / \alpha}}{\int_{T_{0}}^{\delta(t)}\left(\frac{1}{r(s)}\right)^{1 / \alpha} \Delta s}=\frac{1}{\beta(t)} .
$$

Thus, from the last inequality, we get

$$
z(\delta(t)) \geq \beta(t) z^{\sigma}(t)
$$

Hence, using (2.25), we have

$$
\left(\frac{z(\delta(t))}{z^{\sigma}(t)}\right)^{\alpha} \geq\left(\frac{\beta(t) z^{\sigma}(t)}{z^{\sigma}(t)}\right)^{\alpha}=(\beta(t))^{\alpha} .
$$

Applying (2.26) to (2.13), we obtain

$$
w^{\Delta}(t) \leq P_{2}(t)+\frac{\alpha\left(w^{\sigma}(t)\right)^{1+1 / \alpha}}{(r(t))^{1 / \alpha}} .
$$

The rest proof of part (ii) is similar to the proof of part (i). The proof of part (ii) is finished.

The following Lemma will be used in the proof of the next theorems.

Lemma1 $^{[5]}$. Let $\gamma, B \in(0, \infty), A \in R$. Then $F(v)=A v-B v^{\frac{\gamma+1}{\gamma}}$ attains its maximum value on $\mathrm{R}$ such that

$$
\max _{v \in R} F(v)=\frac{\gamma^{\gamma}}{(\gamma+1)^{\alpha+1}} \cdot \frac{A^{\gamma+1}}{B^{\gamma}} .
$$

Theorem2.2. Assume that $\left(H_{1}\right)-\left(H_{3}\right)$ hold. Let $T_{0} \geq t_{0}$. Then there is $a_{0}$ and $b_{0}$ such that $T_{0}<a_{0}<b_{0}$. Let $D\left(a_{0}, b_{0}\right)=\left\{H(t) \in C_{r d}^{1}\left[a_{0}, b_{0}\right]_{\mathrm{T}}: H(t) \neq 0, t \in\left(a_{0}, b_{0}\right)_{\mathrm{T}}, H\left(a_{0}\right)=H\left(b_{0}\right)=0\right\}$.If there are functions $H_{1}(t)$ and $H_{2}(t) \in D\left(a_{0}, b_{0}\right)$ such that

(i) if $\delta(t)>t$ and

$$
\int_{a_{0}}^{b_{0}}\left[H_{1}(s) P_{1}(s)-\frac{r(s)\left(H_{1}^{\Delta}(s)\right)^{\alpha+1}}{(\alpha+1)^{\alpha+1}\left(H_{1}(s)\right)^{\alpha}}\right] \Delta s>0
$$

or

(ii)if $\delta(t) \leq t$ and

$$
\int_{a_{0}}^{b_{0}}\left[H_{2}(s) P_{2}(s)-\frac{r(s)\left(H_{2}^{\Delta}(s)\right)^{\alpha+1}}{(\alpha+1)^{\alpha+1}\left(H_{2}(s)\right)^{\alpha}}\right] \Delta s>0,
$$

then every solution to equation (1.1) is oscillatory on $\left[t_{0}, \infty\right)_{\mathrm{T}}$.

Proof. Suppose not. Without loss of generality, we may suppose that $x(t)$ is an eventually positive solution to equation (1.1). Then there is a $t_{1} \geq t_{0}$ such that $x(t)>0, x(\tau(t))>0$ and $x(g(t, \xi))>0$ for all $t \in\left[t_{1}, \infty\right)_{\mathrm{T}}$ and $\xi \in[a, b]_{\mathrm{T}}$. We proceed as in the proof of Theorem 2.1 until we find (2.20). Multiplying both sides of (2.20) by $H_{1}(t)$ and integrating from $a_{0}$ to $b_{0}$, we obtain 


$$
\int_{a_{0}}^{b_{0}} H_{1}(s) P_{1}(s) \Delta s \leq \int_{a_{0}}^{b_{0}} w^{\sigma}(s) H_{1}^{\Delta}(s) \Delta s-\int_{a_{0}}^{b_{0}} \frac{\alpha H_{1}(s)\left(w^{\sigma}(s)\right)^{1+\frac{1}{\alpha}}}{(r(s))^{1 / \alpha}} \Delta s=\int_{a_{0}}^{b_{0}}\left[w^{\sigma}(s) H_{1}^{\Delta}(s)-\frac{\alpha H_{1}(s)\left(w^{\sigma}(s)\right)^{1+\frac{1}{\alpha}}}{(r(s))^{1 / \alpha}}\right] \Delta s
$$

For simplicity setting $A=H_{1}^{\Delta}(s), B=\frac{\alpha H_{1}(s)}{(r(s))^{1 / \alpha}}, v=w^{\sigma}(t)$, applying Lemma 1 to (2.28), we have

$$
\int_{a_{0}}^{b_{0}}\left[H_{1}(s) P_{1}(s)-\frac{r(s)\left(H^{\Delta}(s)\right)^{\alpha+1}}{(\alpha+1)^{\alpha+1}(H(s))^{\alpha}}\right] \Delta s \leq 0,
$$

which is a contradiction with (2.27). Therefore, the proof of part (i) is complete. Since the proof of part (ii) is similar to the proof of part (i), we omit it here. The proof of this theorem is complete.

Theorem 2.3 Assume that $\left(A_{1}\right)-\left(A_{3}\right)$ hold. In addition, suppose that there is a rd-continuous and $\Delta$-differentiable positive function $\rho(t)$ such that

$$
\lim _{t \rightarrow \infty} \operatorname{Sup} \int_{t_{0}}^{t}\left[\rho(s) Q(s)-\frac{r(\delta(s))\left(\rho_{+}^{\Delta}(s)\right)^{\alpha+1}}{(\alpha+1)^{\alpha+1}\left(\rho(s) \delta^{\Delta}(s)\right)^{\alpha}}\right] \Delta s=\infty .
$$

where $\rho_{+}^{\Delta}(t)=\max \left\{0, \rho^{\Delta}(t)\right\}$. Then every solution of $(1.1)$ is oscillatory on $\left[t_{0}, \infty\right]_{\mathrm{T}}$.

Proof. Suppose not. Without loss of generality, we may assume that $x(t)$ is an eventually positive solution to equation (1.1). We proceed as in the proof of the Theorem 2.1 until we find (2.2) and (2.6). Then using (2.2) and the Potzche chain, we obtain

$$
\begin{aligned}
\left(\mathrm{z}^{\alpha}(\delta(t))\right)^{\Delta} & =\alpha \int_{0}^{1}(z(\delta(t))+h z(t)(z(\tau(t))))^{\alpha-1} d h(z(\delta(t))) \\
& =\alpha \int_{0}^{1}[h z(\delta(\sigma(t)))+(1-h) z(\delta(t))]^{\alpha-1} d h z^{\Delta}(\delta(t)) \delta^{\Delta}(t) \\
& =\alpha z^{\alpha-1}(\delta(t)) z^{\Delta}(\delta(t)) \delta^{\Delta}(t)
\end{aligned}
$$

and

$$
\frac{z^{\Delta}(\delta(t))}{z(\delta(t))} \geq\left(\frac{r(\sigma(t))}{r(\delta(t))}\right)^{\frac{1}{\alpha}} \cdot \frac{z^{\Delta}(\sigma(t))}{z(\delta(\sigma(t)))}
$$

where $\alpha \geq 1$.

Define

$$
y(t)=\rho(t) \frac{r(t)\left(z^{\Delta}(t)\right)^{\alpha}}{z^{\alpha}(\delta(t))} .
$$

Then, clearly, $y(t) \geq 0$ for all $t \in\left[t_{2}, \infty\right]_{\mathrm{T}}$. By using (2.30), (2.31) and Lemma 1, we get

$$
\begin{aligned}
y^{\Delta}(t) & =\rho(t) \frac{\left(r(t)\left(z^{\Delta}(t)\right)^{\alpha}\right)^{\Delta}}{z^{\alpha}(\delta(t))}+\rho^{\Delta}(t) \frac{r(\sigma(t))\left(z^{\Delta}(\sigma(t))\right)^{\alpha}}{z^{\alpha}(\delta(\sigma(t)))}+h(t) r(\sigma(t))\left(z^{\Delta}(\sigma(t))\right)^{\alpha}\left(\frac{1}{z^{\alpha}(\delta(t))}\right)^{\Delta} \\
& \leq-\rho(t) Q(t)+\frac{r(\delta(t))\left(\rho_{+}^{\Delta}(t)\right)^{\alpha+1}}{(\alpha+1)^{\alpha+1}\left(\rho(t) \delta^{\Delta}(t)\right)^{\alpha}} .
\end{aligned}
$$

Integrating (2.33) both sides from $t_{2}$ to $t$, we obtain

$$
y(t)-y\left(t_{2}\right) \leq-\int_{t_{2}}^{t}\left[\rho(s) Q(s)-\frac{r(\delta(s))\left(\rho_{+}^{\Delta}(s)\right)^{\alpha+1}}{(\alpha+1)^{\alpha+1}\left(\rho(s) \delta^{\Delta}(s)\right)^{\alpha}}\right] \Delta s
$$

which implies that

$$
\int_{t_{2}}^{t}\left[\rho(s) Q(s)-\frac{r(\delta(s))\left(\rho_{+}^{\Delta}(s)\right)^{\alpha+1}}{(\alpha+1)^{\alpha+1}\left(\rho(s) \delta^{\Delta}(s)\right)^{\alpha}}\right] \Delta s \leq y\left(t_{2}\right)-y(t) \leq y\left(t_{2}\right) .
$$

This is a contradiction with (2.29). 


\section{Acknowledgement}

This research was supported by science and technology projects foundation of Hengyang (2014KJ22),social science fund project of Hengyang (2014D060),Cultivation of young teachers of Hengyang Normal University of China and Construct program of the key discipline in Hunan Province[2011(76)].

\section{References}

[1] R. P. Agarwal, M. Bohner and S. H. Saker. Os-cillation of second order delay dynamic equations. Can.Appl. Math. G,2005,13, 1-18.

[2] Y. Sahinear. Oscillation of second order delay differential equations on time scales. Nonlinear Anal,2005,63, 1073-1080.

[3] T. Candan. Oscillation of second-order nonlinear neutral dynamic equations on time scales with distributed deviating arguments. Comp. Math. Appl., 2011,62,4118-4125.

[4] S. H. Saler. Oscillation of second order nonlinear neutral delay dynamic equations. Comp.Appl.

Math. 2006, 187,123-141.

[5] S. H. Saker, Oscillation of second order neutral linear differential equations. J. Math. Anal. Appl. ,1995, 194,312-321.

[6] M. Huang and W. Feng. Oscillation for forced second order nonlinear dynamic equations on time scales. Elect. J. Diff. Eqn., 2005, 145, 1-8.

[7] S. H. Saler. Oscillation of superlinear and sub-linear neutral delay dynamic equations. J. Commun.Appl.Anal.2008, 12(2),173-187.

[8] E. Thandapani and V. Piramantham. Oscillation criteria of second-order neutral delay dynamic equations with distributed deviating arguments, Electronic Journal of Qualitative Theory of Dif-ferential Equations, 2010,61, 1-15. 